\title{
INITIAL ROUGHNESS AND RELAXATION BEHAVIOUR OF MBE GROWN ZnSe/GaAs
}

\author{
B. Buda, O. Leifeld, S. Völlmeke, F. Schmilgus, D.J. As, D. Schikora \\ AND K. LISCHKA
}

University of Paderborn, FB Physik, 33098 Paderborn, Germany

\begin{abstract}
We investigated the GaAs/ZnSe interface and the influence of the $\mathrm{Ga}_{2} \mathrm{Se}_{3}$ formation at the $\mathrm{GaAs} / \mathrm{ZnSe}$ interface on the relaxation of the $\mathrm{ZnSe}$ epilayer using reflection high-energy electron diffraction, atomic force microscope, photoluminescence, and X-ray diffraction techniques. An improvement of the surface roughness due to the cleaning of the $\mathrm{GaAs}$ substrate with hydrogen excited in a plasma source and a higher critical thickness of $\mathrm{GaAs}(001) / \mathrm{ZnSe}$ due to the suppression of $\mathrm{Ga}_{2} \mathrm{Se}_{3}$ at the surface was observed.

PACS numbers: 68.55.-a
\end{abstract}

\section{Introduction}

A great deal of attention has been paid to the $\mathrm{ZnSe} / \mathrm{GaAs}$ system after the recent demonstration of molecular beam epitaxy (MBE) grown laser and light emitting diode [1] structures operating in the blue-green region of the visible spectrum. A significant problem with this system, however, is the degeneration of the devices due to the formation of defects. Laser structures grown on GaAs contain a III-V/II-VI heterointerface. The structure of the interface plays a critical role in the formation of defects in the II-VI device. Therefore, research was focused or. detailed studies of the interface structure. One problem is the roughness and the stoichiometry of the GaAs surface. Recent improvements of crystal quality were achieved by using a GaAs buffer layer or by deoxidizing the GaAs substrate with hydrogen plasma instead of the conventional thermal cleaning.

Another problem is the formation of $\mathrm{Ga}_{2} \mathrm{Se}_{3}$ at the interface due to the exchange of As and Se forced by different formation enthalpies [2]. $\mathrm{Ga}_{2} \mathrm{Se}_{3}$ causes an initial 3D island growth mode [3] which influences the generation of dislocations and the structural quality of the epilayer. Until now this influence is not fully understood.

In this paper we study the roughness of the GaAs substrate depending on the preparation procedure before growth. For the GaAs cleaning we used excited hydrogen from a plasma source and different substrate temperatures. We studied the effect on the structural quality of the $\mathrm{ZnSe} / \mathrm{GaAs}$ epilayer. We also show a dependence of the relaxation behaviour of the $\mathrm{ZnSe}$ epilayer on the formation of $\mathrm{Ga}_{2} \mathrm{Se}_{3}$ at the heterointerface. 


\section{Experiments}

The experiments were performed in an MBE system equipped with a reflection high-energy electron diffraction system (RHEED-system) and a rf (13.56 MHz) plasma discharge, free-radical source manufactured by Oxford Applied Research. The rf-source was used either as a nitrogen source for $p$-type doping of $\mathrm{ZnSe}$ or as a source for hydrogen radical beam to clean the GaAs substrate. The two gases were cleaned separately with purifiers to an order of $8 \mathrm{~N}$. To study the influence of the substrate preparation on the surface roughness and the crystal quality, epilayers of $\mathrm{ZnSe}$ with different thicknesses were grown at a growth temperature of $325^{\circ} \mathrm{C}$. We employed a Se to $\mathrm{Zn}$ beam equivalent pressure ratio of 2.2. A series of $200 \mathrm{~nm}$ thick $\mathrm{ZnSe}$ epilayers were grown on epiready GaAs (001) substrates. One GaAs substrate was cleaned by conventional thermal treatment at $610^{\circ} \mathrm{C}$, the other ones under hydrogen plasma at temperatures between $400^{\circ} \mathrm{C}$ and $590^{\circ} \mathrm{C}$ for about $10 \mathrm{~min}$. All other GaAs substrates were cleaned at $400^{\circ} \mathrm{C}$. The background pressure of hydrogen in the MBE system was about $5 \times 10^{-6} \mathrm{mbar}$ and the power used to create the discharge was $450 \mathrm{~W}$. To investigate the influence of the formation of $\mathrm{Ga}_{2} \mathrm{Se}_{3}$ at the GaAs surface on the relaxation of the $\mathrm{ZnSe}$ epilayer, we cleaned the GaAs substrate by hydrogen with and without an additional $\mathrm{Zn}$ flux during deoxidation. The surfaces of the growing ZnSe layer was observed by RHEED and the FWHM of the specular spot perpendicular to the shadow edge was measured. The post growth surface roughness was determined by atomic force microscope (AFM) in contact mode. The X-ray diffraction measurements ((004)-reflex rocking curves) were performed with a Philips MRD diffractometer equipped with a four-crystal Bartels X-ray monochromator yielding a highly monochromatic $\left(\Delta \lambda / \lambda=10^{-5}\right)$ primary beam with low divergence (about 10 arcsec). The photoluminescence measurements were performed at a temperature of $2 \mathrm{~K}$. The samples were excited by an $\mathrm{HeCd}$-laser $(325 \mathrm{~nm})$ with an intensity of $0.25 \mathrm{~W} / \mathrm{cm}^{2}$.

\section{Results and discussion}

The crystal quality and the dislocation density in ZnSe epilayers depend on the roughness and stoichiometry of the GaAs substrate. From AFM measurements we find that the surface roughness of the growing epilayer is strongly correlated to the roughness of the GaAs substrate. Thus it is important to have an atomic smooth GaAs surface. By comparing the AFM roughness of thermally cleaned GaAs surfaces with those of substrates cleaned by excited hydrogen, we find an improvement of the surface smoothness by one order of magnitude. This result is confirmed by RHEED measurements. Because of the chemical reaction of hydrogen radicals with GaAs oxide and a higher vapour pressure of the resulting chemical products such as $\mathrm{Ga}-\mathrm{H}, \mathrm{As}-\mathrm{H}$ and $\mathrm{H}_{2} \mathrm{O}$, a lower processing temperature of about $400^{\circ} \mathrm{C}$ can be used for complete cleaning of the substrate. Due to the lower processing temperature, the As depletion is reduced and the GaAs surface is smoother on the atomic scale.

To confirm this result we investigated $\mathrm{ZnSe}$ epilayers of $200 \mathrm{~nm}$ thickness grown on GaAs substrates cleaned at various temperatures. We observed that 
ZnSe layers grown on substrates cleaned by hydrogen at low temperatures reveal a smaller FWHM of X-ray rocking curves. The FWHM decreases from 900 arcsec for the thermally cleaned $\left(610^{\circ} \mathrm{C}\right) \mathrm{GaAs}$ to about 95 arcsec for GaAs cleaned at $400^{\circ} \mathrm{C}$. These results are confirmed by photoluminescence measurements.

Figure 1 shows the photoluminescence spectra of the thermally deoxidized and the hydrogen treated samples. A clear decrease in the defect related luminescence intensity (SA and Y-line) relative to the free exciton intensity is seen for the hydrogen treated samples with decreasing cleaning temperature. Another reason for a high dislocation density in the $\mathrm{ZnSe}$ layer may be the formation of $\mathrm{Ga}_{2} \mathrm{Se}_{3}$ at the $\mathrm{GaAs} / \mathrm{ZnSe}$ interface. The formation of cubic $\mathrm{Ga}_{2} \mathrm{Se}_{3}$ is forced by the exchange of As and $\mathrm{Se}$ at the GaAs surface. $\mathrm{Ga}_{2} \mathrm{Se}_{3}$ at the surface can be detected by RIIEED because of its $(2 \times 1)$ reconstruction [4].

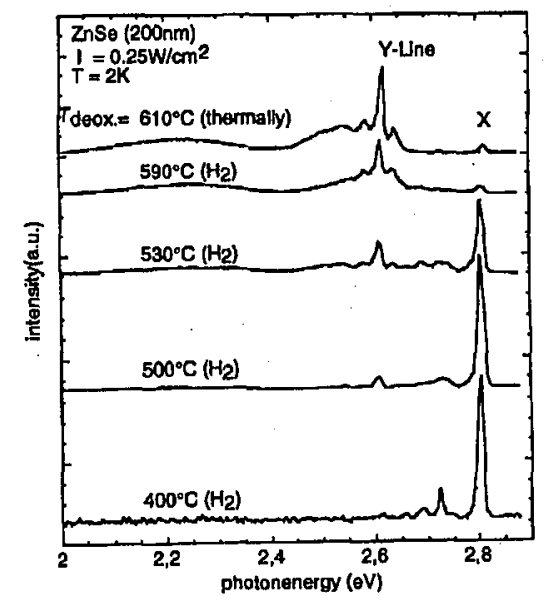

Fig. 1. Photoluminescence spectra of $200 \mathrm{~nm}$ thick $\mathrm{ZnSe}$ epilayers grown on GaAs(001) substrates thermally cleaned at $T_{\text {deox. }}=610^{\circ} \mathrm{C}$ and $\mathrm{H}_{2}$-plasma treated at various temperatures.

When the GaAs substrate is cleaned by hydrogen under Zn-partial pressure, the formation of $\mathrm{Ga}_{2} \mathrm{Se}_{3}$ on the surface is suppressed as shown in Fig. 2. RHEED patterns indicate that $\mathrm{ZnSe}$ grow in a 3D island growth mode when the initial GaAs surface shows a clear $(2 \times 1)$ surface reconstruction. However, a 2D growth mode is observed already after a few seconds when ZnSe growth starts on a nearly unreconstructed GaAs surface.

Figure 3 shows the FWHM of the specular spot RHEED reflex measured perpendicular to the shadow edge as a function of the ZnSe layer thickness. The observed pronounced increase in the FWHM is due to the onset of the lattice relaxation. A critical thickness of $225 \mathrm{~nm}$ was observed for layers with a 3D nucleation in agreement with results from other groups [5]. In case of a nearly $2 \mathrm{D}$ initial growth mode a critical thickness $h_{\mathrm{c}}$ of $280 \mathrm{~nm}$ was measured. Using transmission electron microscopy (TEM), it has been shown in Ref. [6] that the dominant defect configuration is different in the samples with and without the formation of 


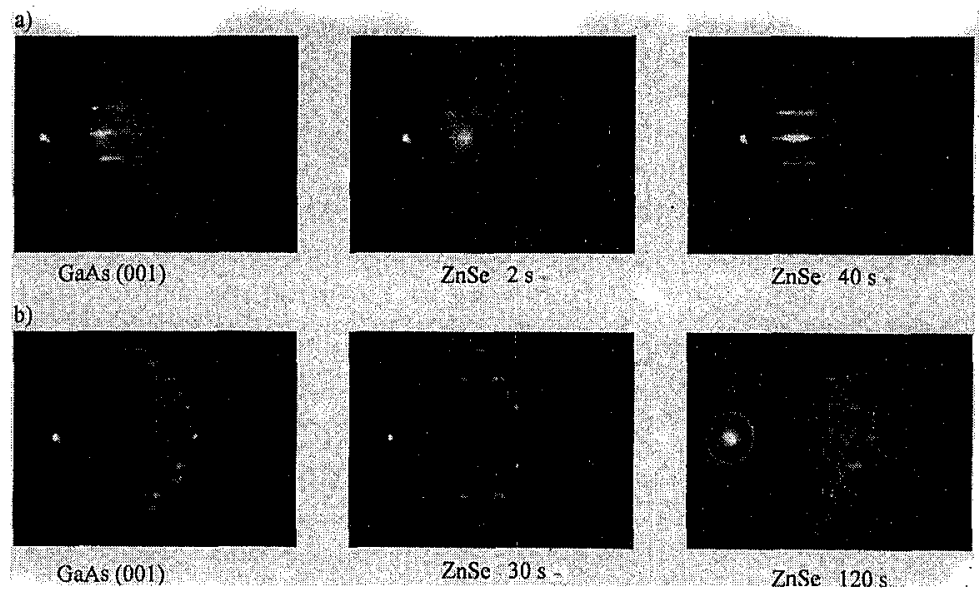

Fig. 2. RHEED patterns of the nucleation phase of $\mathrm{ZnSe}$ on $\mathrm{GaAs}(001)$ taken in the [110] azimuth. GaAs substrates cleaned with hydrogen and (a) with additional $\mathrm{Zn}$ flux, (b) without $\mathrm{Zn}$ flux. The substrate temperature was $400^{\circ} \mathrm{C}$.

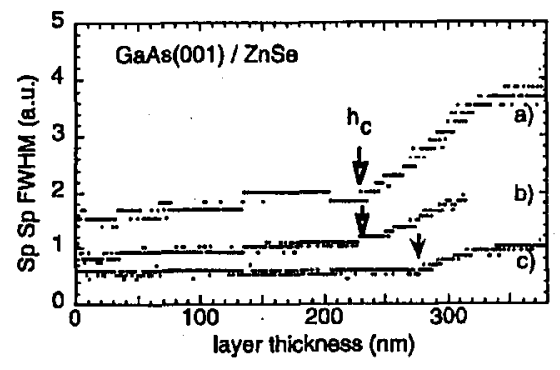

Fig. 3. The FWHM of the specular spot perpendicular to the shadow edge in [1-10] azimuth. ZnSe layers are grown on $a-\mathrm{GaAs}(001)$ surface cleaned by excited hydrogen, $b-\mathrm{GaAs}(001)$ surface $2^{\circ}$ off (111) cleaned by excited hydrogen, $c-\mathrm{GaAs}(001)$ surface cleaned by excited hydrogen and additional $\mathrm{Zn}$ flux.

$\mathrm{Ga}_{2} \mathrm{Se}_{3}$ at the interface. The different activation energies of these defects may lead to different critical thicknesses. Another reason for the variation of $h_{\mathrm{c}}$ may be the different lattice constants of GaAs (5.65 $\AA$ ) and $\mathrm{Ga}_{2} \mathrm{Se}_{3}(5.43 \AA$ ) [4] yielding a decreased lattice misfit between the substrate and the $\mathrm{ZnSe}$ layer when the formation of $\mathrm{Ga}_{2} \mathrm{Se}_{3}$ interface layer is suppressed.

\section{Conclusion}

We found an improved crystalline quality of $\mathrm{ZnSe}$ epilayer when excited hydrogen is used to clean the GaAs surface. We also found that the roughness of the growing surface is mainly determined by the roughness of the GaAs substrate. Further we detected an increase in the critical thickness due to the possible suppression of $\mathrm{Ga}_{2} \mathrm{Se}_{3}$ formation at the interface. 


\section{Acknowledgments}

This work was supported by "Deutsche Forschungsgemeinschaft" (DFG).

\section{References}

[1] M.A. Haase, J. Qui, J.M. DePuydt, H. Cheng, Appl. Phys. Lett. 59, 1272 (1991).

[2] A. Krost, W. Richter. D.R.T. Zahn, K. Hingerl, H. Sitter, Appl. Phys. Lett. 57, 1981 (1990).

[3] D. Li, M.D. Pashley, J. Vac. Sci. Technol. B 12, 2547 (1994).

[4] S. Takatani, T. Kikawa, M. Nakazawa, Phys. Rev. B 45, 8498 (1992).

[5] T. Reisinger, M.J. Kaster, K. Wolf, E. Steinkirchner, W. Hückl, H. Stanzl, W. Gebhardt, Mater. Sci. Forum 182, 147 (1995).

[6] S. Guha, H. Munekata, L.L. Chang, J. Appl. Phys. 73, 2294 (1993). 